PSICOLOGIA, SAÚDE \& DOENÇAS, 2019, 20(2), 300-318

ISSN - 2182-8407

Sociedade Portuguesa de Psicologia da Saúde - SPPS - www.sp-ps.pt

DOI: http://dx.doi.org/10.15309/19psd200203

\title{
PREVALÊNCIA E FATORES ASSOCIADOS À SÍNDROME DE BURNOUT ENTRE UNIVERSITÁRIOS: REVISÃO DE LITERATURA
}

\author{
Gabrielle Moura ${ }^{1}$, Maria Brito ${ }^{1}$, Lucinéia Pinho $^{1}$, Viviane Reis ${ }^{1}$, Luís Souza ${ }^{2}, \&$ Tatiana Magalhães ${ }^{3}$ \\ ${ }^{1}$ Universidade Estadual de Montes Claros, Centro de Ciências Biológicas e da Saúde, Unimontes, Montes Claros- \\ Minas Gerais, Brasil, gabymoura4@hotmail.com,mfsfbrito@yahoo.com.br, lucineiapinho@hotmail.com, \\ viola.chaves@yahoo.com.br \\ ${ }^{2}$ Universidade Federal de São Joao del Rei, UFSJ. Universidade do Vale do Rio Verde, UniCor, Betim e Belo \\ Horizonte, Brasil, luis.pauloss@hotmail.com \\ ${ }^{3}$ Secretaria Municipal de Saúde, Núcleo de Atenção Primária à Saúde - Montes Claros- Minas Gerais, Brasil, \\ tatimagmoc@gmail.com
}

RESUMO: Este estudo objetivou identificar as prevalências e os fatores associados à Síndrome de Burnout (SB) entre universitários, a partir da análise das publicações. Trata-se de uma revisão da literatura, realizada em janeiro de 2018, nas bases LILACS, MEDLINE e SciELO. Consideraram-se artigos publicados entre 2006 e 2017, em português, inglês ou espanhol. Foram incluídos 15 artigos nesta, sendo a maior parte conduzida no Brasil e publicada em 2012. As prevalências gerais da SB variaram de $2,5 \%$ a $57,2 \%$. Entre as dimensões, as prevalências variaram de $30,43 \%$ a $67 \%$ para a exaustão emocional; $41,4 \%$ a $68,9 \%$ para descrença ou cinismo; $17,3 \%$ a $45,7 \%$ para eficácia profissional. Entre os fatores associados, destacaram: sexo, idade, tipo de moradia, falta de tempo de lazer, inscrição em um maior número de disciplinas, insatisfação com o curso, semestres avançados, consumo de medicações devido aos estudos. Os fatores associados estiveram, em sua maioria, envolvidos com questões diretamente relacionadas à vida acadêmica. Considerar os universitários como um grupo importante dentro do contexto da saúde ocupacional é muito importante, uma vez que o início do Burnout pode ocorrer no período acadêmico e persistir na vida profissional.

Palavras-chave: burnout, universitários, estudantes, esgotamento profissional.

\section{PREVALENCE AND FACTORS ASSOCIATED WITH BURNOUT SYNDROME AMONG UNIVERSITY STUDENTS: A LITERATURE REVIEW}

ABSTRACT: This study aimed to identify the prevalences and factors associated with Burnout Syndrome (SB) among university students, based on the analysis of the publications. Methods: This is a review of the literature, conducted in January 2018, in the LILACS, MEDLINE and SciELO databases. Articles published between 2006 and 2017 were considered in Portuguese, English or Spanish. 15 articles were included in this study, most of which were conducted in Brazil and published in 2012. The overall prevalence of SB varied from $2.5 \%$ to $57.2 \%$. Among the dimensions, the prevalence ranged from $30.43 \%$ to $67 \%$ for emotional exhaustion; $41.4 \%$ to $68.9 \%$ for disbelief or cynicism; $17.3 \%$ to $45.7 \%$ for professional effectiveness. Among the associated factors

Rua Coronel Francisco Jose Souto, Número 160, Bairro Todos os Santos, Montes Claros- MG, Brasil. Tel: (38) 99238-8328. email: gabymoura4@gmail.com. 
were: sex, age, type of housing, lack of leisure time, enrollment in a greater number of subjects, dissatisfaction with the course, advanced semesters, consumption of medications due to studies. The associated factors were, for the most part, involved with issues directly related to academic life. Considering university students as an important group within the context of occupational health is very important, since the onset of Burnout can occur in the academic period and persist in working life.

Keywords: burnout, college students, students, professional exhaustion.

Recebido em 28 de Agosto de 2018/ Aceite em 02 de Junho de 2019

A universidade tem como missão a formação integral dos seus alunos, contribuindo para a construção da humanidade, visto que o conhecimento é o ponto primordial para o melhor desenvolvimento humano. Por outro lado, a graduação pode ter um peso negativo sobre os alunos, já que os mesmos passam por grandes momentos de estresse devido às cobranças, às constantes avaliações, às pressões do tempo, aos estágios e, às vezes, pelo anseio da insatisfação com o curso escolhido (Sobrinho, 2014; Tomaschewski-Barlem et al., 2014)

A presença constante de sentimentos estressores e negativos podem ser sinais do desenvolvimento da Síndrome de Burnout (SB), que é uma síndrome psicossocial caracterizada pela exaustão emocional, que acomete com maior frequência indivíduos que, de alguma maneira, lidam com outras pessoas. O impacto da SB é evidenciado em estudos nacionais e internacionais, demonstrando os sinalizadores do Burnout nos alunos em fase de formação profissional e não mais unicamente nos profissionais (Campos, Jordani, Zucoloto, Bonafé, \& Maroco, 2012; Carlotto, Nakamura, \& Câmara, 2006; Chagas, Junior, Cunha, Caixeta, \& Fonseca, 2016; Maslach \& Jackson, 1981; Peleias, Guimarães, Chan, \& Carlotto, 2017; Vilela, Pacheco, \& Carlos, 2013).

O Burnout em estudantes é constituído por três dimensões bem definidas: exaustão emocional caracterizada pelo desgaste vivido no cotidiano do curso (cansaço físico e mental, desânimo, estresse, irritabilidade, cefaleias, dores musculares e alterações no sono); descrença ou ceticismo definido pelas manifestações de comportamento defensivo, em que o indivíduo passa a ausentar-se das aulas, diminuindo o contato com professores e colegas; eficácia profissional - esta engloba os sentimentos de baixa autoestima, inferioridade, insuficiência, impotência e insegurança, aumentando o desagrado com o curso (Tomaschewski-Barlem et al., 2013).

Ao analisar estudos sobre a SB em estudantes, verifica-se a influência de certos fatores sobre este agravo, sendo os principais: não morar com os pais ou familiares; não estar em um relacionamento amoroso; não praticar atividades físicas ou de lazer; não estar no curso de primeira escolha no vestibular; estar em um trabalho extracurricular; ter vontade de desistir do curso; possuir distúrbios mentais (depressão, ansiedade, entre outros) (Christofoletti, Trelha, Galera, \& Feracin, 2007; Goni, Danza, Urgoiti, \& Durante, 2015).

A literatura aponta, ainda, que ao vivenciarem a SB, os alunos podem sofrem consequências negativas nos níveis individual, profissional, social e familiar, o que exerce influência direta no processo de ensino-aprendizagem. Desta forma, novos estudos são importantes para auxiliarem na prevenção da SB desde a formação acadêmica, evitando o seu agravo e suas repercussões sintomatológicas e comportamentais nas diferentes realidades educacionais do mundo (Azevedo et al., 2012; Campos et al., 2013; Ricardo \& Paneque, 2013).

O objetivo deste estudo foi identificar as prevalências da Síndrome de Burnout entre universitários, assim como os fatores que associam a este desfecho, a partir da análise das publicações da última década. 


\section{PREVALÊNCIA DE BURNOUT ENTRE UNIVERSITÁRIOS}

MÉTODO

Trata-se de uma revisão da literatura, realizada conforme recomendações metodológicas do protocolo Preferred Reporting Items for Systematic Reviews and Meta-Analyses (PRISMA) (Liberati, 2009). O processo de elaboração desta revisão foi iniciado com a formulação de questões de pesquisa que apresentassem relevância para a saúde pública e ocupacional: Qual a prevalência da Síndrome de Burnout entre universitários, em nível nacional e internacional? Quais fatores estão envolvidos na ocorrência da SB neste grupo populacional?

\section{Estratégias de busca}

As buscas ocorreram em janeiro de 2018, tendo sido realizada por mais de um autor, para que houvesse conferência das informações colhidas em todas as etapas.

Realizaram-se buscas online em bases vinculadas à Biblioteca Virtual em Saúde (BVS) Literatura Latino-Americana e do Caribe em Ciências da Saúde (LILACS) e Medical Literature Analysis and Retrieval System Online (MEDLINE); e no Scientific Electronic Library Online (SciELO). Estas bases foram escolhidas pelos autores por entenderem que abrangem a literatura publicada nos diversos países do mundo e incluem periódicos conceituados da área da saúde.

Como descritores, foram utilizados os listados nos Descritores de Ciências em Saúde (DeCS) e no Medical Subject Headings (MeSH), sendo os seguintes: "Burnout", "síndrome de Burnout", "esgotamento", "agotamiento", "estudantes"; "alunos", "acadêmicos", "universitários", "alumno". Os descritores do MeSH utilizados foram: "Burnout", "Burnout syndrome", "exhaustion", "student", "academic". Destaca-se, ainda, que foram utilizados o "AND" e o "OR" como operadores booleanos.

Nas bases LILACS e MEDLINE, foram adotadas as seguintes chaves de buscas: (Burnout) AND (estudantes OR alunos). Na base de dados SciELO, a chave de busca foi: (Burnout OR Burnout syndrome OR exhaustion OR agotamiento) AND (student OR academic OR alunos OR aluno OR universitários).

\section{Critério de inclusão e procedimentos de coleta}

Como critérios de inclusão, levaram-se em consideração os seguintes: artigos originais desenvolvidos com estudantes universitários, com metodologia quantitativa e com uma descrição clara das análises estatísticas realizadas; artigos que utilizassem como ferramenta padrão-ouro o Maslach Burnout Inventory-Student Survey (MBI-SS); publicados no período de 2006 a 2017, em português, inglês ou espanhol; disponíveis na íntegra eletronicamente. Avaliou-se, também, a duplicidade dos artigos, tendo sido considerada somente uma versão. Foram excluídos os relatórios técnicos, as teses e as dissertações, além dos estudos distintos conduzidos na mesma população.

Definidas as estratégias de busca, efetuaram-se as pesquisas. Os artigos foram inicialmente submetidos aos critérios de inclusão, tendo sido identificados 183 trabalhos. Após esta etapa, a seleção dos artigos se deu pela leitura exploratória do título e resumo de cada referência. Em alguns artigos, optou-se por fazer uma análise prévia, procedendo a uma leitura na íntegra, para uma melhor definição de inclusão ou exclusão. Assim, 125 (68 \%) foram excluídos por duplicidade e/ou por não atenderem estritamente a temática/desenho de estudo. Dos 58 restantes, todos foram lidos na íntegra. Nesta etapa, 35 foram excluídos por não utilizarem o MBI-SS como instrumento padrãoouro e/ou por não apresentarem a descrição da análise estatística e 8 foram excluídos por serem estudos distintos conduzidos na mesma população. Dessa maneira, compuseram a amostra final desta revisão 15 artigos (Figura 1). 


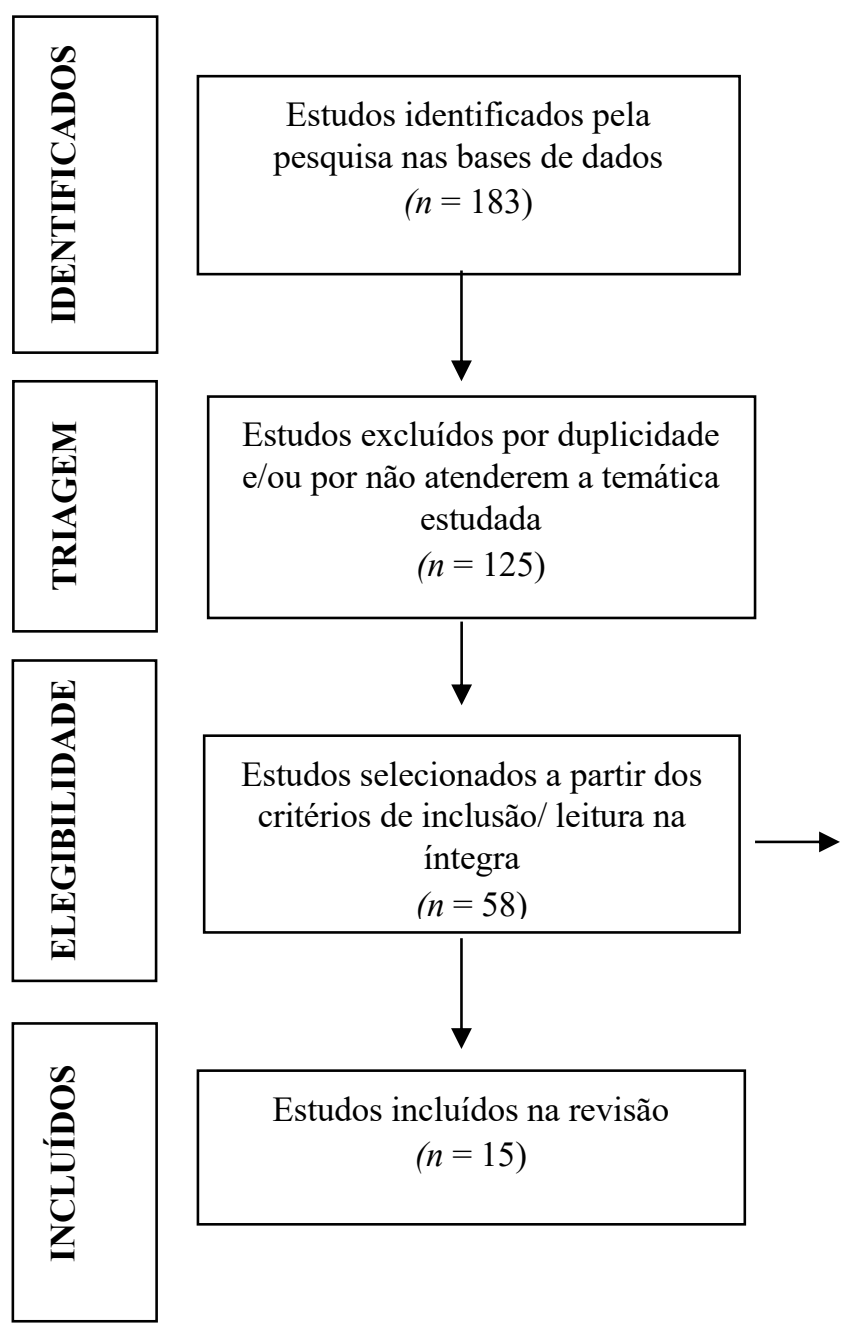

\section{Excluídos nesta etapa:}

- 35 não utilizarem o Maslach Burnout Inventory-Student Survey (MBI-SS) como padrão-ouro e/ou não descreveram as análises estatísticas.

- 8 Estudos distintos conduzidos na mesma população.

\section{Figura 1.}

Representação esquemática das etapas de identificação, triagem, elegibilidade e inclusão de trabalhos na revisão, 2006-2017.

A etapa de seleção dos artigos foi realizada de forma independente por dois pesquisadores, e as discordâncias entre eles resolvidas mediante discussão e consenso de um terceiro avaliador.

\section{Análise dos dados e avaliação da qualidade dos estudos}

A avaliação da qualidade dos artigos se deu conforme os critérios metodológicos propostos pelo Checklist for Measuring Quality (Downsm \& Black, 1998), instrumento utilizado para a avaliação e pontuação de artigos selecionados em revisões de literatura. A versão original deste instrumento é composta de 27 itens, contudo, para este estudo, adotou-se a versão reduzida de 17 itens que avaliam estudos observacionais, sendo excluídos 10 itens relacionados a estudos experimentais. $\mathrm{O}$ instrumento contempla as questões: "Os objetivos foram claramente descritos? Desfecho claramente descrito na introdução ou metodologia? O estudo apresentou as características dos participantes incluídos? Houve distribuição das principais variáveis de confusão? Principais resultados claramente descritos? Foi oferecida informação sobre estimativas de variabilidade aleatória nos dados? Características das perdas foram apresentadas? Os valores reais de probabilidade foram reportados para os resultados principais? Houve representatividade dos indivíduos incluídos nos estudos? Foi deixado de forma clara pelos autores algum resultado que não tenha sido baseado em 


\section{PREVALÊNCIA DE BURNOUT ENTRE UNIVERSITÁRIOS}

hipótese estabelecida anteriormente pela pesquisa? Se em estudos de coorte, a análise foi ajustada para diferentes durações de acompanhamento ou se em estudo de casos e controles o tempo de intervenção foi o mesmo para casos e controles? Adequação dos testes estatísticos? As medidas utilizadas foram acuradas? Os participantes dos diferentes grupos foram recrutados na mesma população? Os participantes dos diferentes grupos foram recrutados no mesmo período de tempo? A análise incluiu ajuste adequado para as variáveis de confusão? Foram consideradas as perdas de participantes durante o estudo?".

Para cada questão, o escore atribuído pode variar de 0 a 1, caso o artigo atenda aos critérios recomendados, com exceção da questão 5 , que pode variar de 0 a 2 . Assim, a pontuação total do instrumento foi de 18 pontos, e os estudos selecionados que apresentaram a classificação mínima de $70 \%$ (12 pontos), foram considerados com alto rigor metodológico e incluídos no estudo. A saber, esses mesmos critérios foram utilizados por outros autores em estudos que analisaram a qualidade de artigos em revisões de literatura (Araújo Vilarim, Sabroza, \& Nardi, 2010; Rossi \& Vasconcelos, 2010; Thiengo, Santos, Mason, Abelha, \& Lovisi, 2011).

Os dados extraídos foram registrados em uma planilha no Microsoft Word $2010^{\circledR}$, dispostos da seguinte maneira: autores e ano de publicação, título, periódico e origem da pesquisa, delineamento de estudo e tamanho da amostra, curso de graduação pesquisado, instrumento de pesquisa, escore da análise de qualidade dos estudos. Registrou-se, ainda, o tipo de análise dos dados, prevalência/média, fatores associados e principais resultados e conclusões. A sumarização das informações coletadas foi organizada em tabelas.

\section{RESULTADOS}

Criou-se um quadro sinóptico que contemplou os aspectos considerados pertinentes nos 15 estudos (Quadro 1). 
Gabrielle Moura, Maria Brito, Lucinéia Pinho, Viviane Reis, Luiz Souza, \& Tatiana Magalhães

Quadro 1.

Características dos artigos incluídos na revisão, 2006 a 2017.

\begin{tabular}{|c|c|c|c|c|c|c|c|}
\hline $\begin{array}{l}\text { Autores (ano de } \\
\text { publicação) }\end{array}$ & Título do artigo & Periódico & $\begin{array}{l}\text { Origem } \\
\text { da } \\
\text { pesquisa }\end{array}$ & $\begin{array}{l}\text { Tipo de } \\
\text { estudo }\end{array}$ & $n$ & $\begin{array}{l}\text { Curso (s) de Graduação } \\
\text { estudado (s) }\end{array}$ & $\begin{array}{c}\text { Escore } \\
\text { análise } \\
\text { qualidade } \\
\text { dos } \\
\text { estudos }\end{array}$ \\
\hline $\begin{array}{l}\text { Carlotto, Nakamura, \& } \\
\text { Câmara, } 2006\end{array}$ & $\begin{array}{l}\text { Síndrome de Burnout em estudantes } \\
\text { universitários da área da saúde }\end{array}$ & Psico & Brasil & Transversal & 514 & $\begin{array}{c}\text { Psicologia, Enfermagem, } \\
\text { Medicina, Odontologia, } \\
\text { Farmácia, Biomedicina, } \\
\text { Fonoaudiologia, } \\
\text { Fisioterapia }\end{array}$ & 14 \\
\hline $\begin{array}{c}\text { Carlotto, } \\
\text { Câmara, Otto, } \\
\text { \& Kauffmann, 2010 }\end{array}$ & $\begin{array}{l}\text { Síndrome de Burnout e coping em } \\
\text { estudantes de psicólogia }\end{array}$ & $\begin{array}{l}\text { Boletim de } \\
\text { Psicologia }\end{array}$ & Brasil & Transversal & 239 & Psicologia & 13 \\
\hline $\begin{array}{c}\text { Estela-Villa, Jiménez- } \\
\text { Román, Landeo- } \\
\text { Gutiérrez, Tomateo- } \\
\text { Torvisco, \& Vega- } \\
\text { Dienstmaier, } 2010 \\
\end{array}$ & $\begin{array}{l}\text { Prevalência de síndrome de Burnout } \\
\text { em alunos del séptimo año de } \\
\text { medicina de una universidad privada } \\
\text { de Lima, Perú }\end{array}$ & $\begin{array}{l}\text { Revista de Neuro- } \\
\text { Psiquiatría }\end{array}$ & Peru & Transversal & 117 & Medicina & 15 \\
\hline $\begin{array}{l}\text { Schulke, Tarouco, } \\
\text { Aloísio, \& Carlotto, } \\
2011\end{array}$ & $\begin{array}{l}\text { El Síndrome de Burnout em } \\
\text { losalumnos } \\
\text { enprácticas de fisioterapia }\end{array}$ & $\begin{array}{l}\text { Diversitas: } \\
\text { Perspectivas en } \\
\text { Psicología }\end{array}$ & Brasil & Transversal & 101 & Fisioterapia & 14 \\
\hline $\begin{array}{l}\text { Campos, Jordani, } \\
\text { Zucoloto, Bonafé, \& } \\
\text { Maroco, } 2012\end{array}$ & $\begin{array}{l}\text { Síndrome de Burnout em graduandos } \\
\text { de Odontologia }\end{array}$ & $\begin{array}{l}\text { Revista Brasileira } \\
\text { de Epidemiologia }\end{array}$ & Brasil & Transversal & 300 & Odontologia & 14 \\
\hline $\begin{array}{l}\text { Costa, Santos, Santos, } \\
\text { Melo, \& Andrade, } 2012\end{array}$ & $\begin{array}{l}\text { Burnout Syndrome and associated } \\
\text { factors among medical students: a } \\
\text { cross-sectional study }\end{array}$ & Clinical Science & Brasil & Transversal & 369 & Medicina & 14 \\
\hline Fogaça et al., 2012 & $\begin{array}{c}\text { Burnout em estudantes de psicologia: } \\
\text { diferenças entre alunos } \\
\text { iniciantes e concluintes }\end{array}$ & Aletheia & Brasil & Transversal & 282 & Psicologia & 13 \\
\hline $\begin{array}{l}\text { Mori, Valentell, \& } \\
\text { Nascimento, } 2012\end{array}$ & $\begin{array}{l}\text { Síndrome de Burnout e rendimento } \\
\text { acadêmico em estudantes da primeira } \\
\text { à quarta série de um curso de } \\
\text { graduação em medicina }\end{array}$ & $\begin{array}{l}\text { Revista Brasileira } \\
\text { de Educação } \\
\text { Médica }\end{array}$ & Brasil & Transversal & 225 & Medicina & 13 \\
\hline
\end{tabular}


PREVALÊNCIA DE BURNOUT ENTRE UNIVERSITÁRIOS

\begin{tabular}{|c|c|c|c|c|c|c|c|}
\hline $\begin{array}{l}\text { Vilela, Pacheco, \& } \\
\text { Carlos, } 2013\end{array}$ & $\begin{array}{l}\text { Síndrome de Burnout e estresse em } \\
\text { graduandos de enfermagem }\end{array}$ & $\begin{array}{c}\text { Revista de } \\
\text { Enfermagem do } \\
\text { Centro Oeste } \\
\text { Mineiro } \\
\end{array}$ & Brasil & Transversal & 118 & Enfermagem & 13 \\
\hline $\begin{array}{c}\text { Tomaschewski-Barlem } \\
\text { et al., } 2014\end{array}$ & $\begin{array}{c}\text { Burnout syndrome among } \\
\text { undergraduate nursing students at a } \\
\text { public university }\end{array}$ & $\begin{array}{l}\text { Revista Latino- } \\
\text { Americana de } \\
\text { Enfermagem }\end{array}$ & Brasil & Transversal & 168 & Enfermagem & 14 \\
\hline Picasso-Pozo, 2015 & $\begin{array}{c}\text { Síndrome de Burnout enestudiantes } \\
\text { de odontología de una universidad } \\
\text { peruana }\end{array}$ & Revista Kiru & Peru & Transversal & 140 & Odontologia & 14 \\
\hline Gomez et al., 2015 & $\begin{array}{l}\text { Relación entre elbienestar y } \\
\text { elrendimiento académico enalumnos } \\
\text { de primer año de medicina }\end{array}$ & $\begin{array}{l}\text { Revista Medica del } \\
\text { Chile }\end{array}$ & Chile & Transversal & 277 & Medicina & 14 \\
\hline $\begin{array}{l}\text { Oliveira, Zucoloto, \& } \\
\quad \text { Campos, } 2015\end{array}$ & $\begin{array}{c}\text { Síndrome de Burnoutem estudantes } \\
\text { de Farmácia-Bioquímica: um estudo } \\
\text { transversal }\end{array}$ & $\begin{array}{l}\text { Revista Brasileira } \\
\text { de Pesquisa em } \\
\text { Saúde } \\
\end{array}$ & Brasil & Transversal & 363 & Farmácia-Bioquímica & 14 \\
\hline $\begin{array}{l}\text { Chagas, Junior, Cunha, } \\
\text { Caixeta, \& Fonseca, } \\
2016\end{array}$ & $\begin{array}{l}\text { Ocorrência da Síndrome de Burnout } \\
\text { em acadêmicos de medicina de } \\
\text { instituição de ensino no interior de } \\
\text { Minas Gerais }\end{array}$ & $\begin{array}{c}\text { Revista de } \\
\text { Medicina e } \\
\text { Saúde de Brasília }\end{array}$ & Brasil & Transversal & 342 & Medicina & 14 \\
\hline $\begin{array}{l}\text { Almeida, Souza, } \\
\text { Almeida, Almeida, \& } \\
\text { Almeida, } 2016\end{array}$ & $\begin{array}{l}\text { The prevalence of Burnout syndrome } \\
\text { in medical students }\end{array}$ & $\begin{array}{c}\text { Archives of } \\
\text { Clinical Psychiatry }\end{array}$ & Brasil & Transversal & 376 & Medicina & 16 \\
\hline
\end{tabular}


Houve um predomínio das publicações com cinco autores. Dos artigos analisados, $10(66,6)$ eram da área de epidemiologia/saúde coletiva e $5(33,3 \%)$ foram publicados em revistas da área de psicologia e educação. Maior parte dos estudos foi conduzida no Brasil (81,2\%), publicada em 2012 $(26,6 \%)$. Os estudos com maiores amostras eram brasileiros, sendo que um analisou 514 acadêmicos dos cursos da área da saúde (Psicologia, Enfermagem, Medicina, Odontologia, Farmácia, Biomedicina, Fonoaudiologia, Fisioterapia) de uma instituição universitária privada da região metropolitana de Porto Alegre, Rio Grande do Sul, e o outro avaliou 376 universitários em Fortaleza, Ceará (Almeida, Souza, Almeida, Almeida, \& Almeida, 2016; Carlotto, Nakamura, \& Câmara, 2006).

Em relação às prevalências e fatores associados à $\mathrm{SB}$, criou-se um quadro com estas informações (Quadro 2). Foi possível identificar que, do total de estudos, 6 (40\%) apresentaram seus resultados na forma de frequência geral (SB geral) ou dimensões em forma de prevalência de acometimento da síndrome; e $9(60 \%)$ apresentaram a média dos escores das dimensões da SB (EE: exaustão emocional; DE: descrença; EP: eficácia profissional). A prevalência geral da síndrome variou de $2,5 \%$ a $57,2 \%$ entre os universitários pesquisados. Entre as dimensões, as prevalências variaram de $30,43 \%$ a $67 \%$ para a exaustão emocional; de 41,4\% a 68,9\% para descrença ou cinismo; $17,3 \%$ a $45,7 \%$ para eficácia profissional. Para os estudos que apresentaram as médias, a variação encontrada foi de 0,84 a 86,66 para exaustão emocional; 0,71 a 79,87 para descrença ou cinismo; 0,65 a 52,88 para eficácia profissional. Aqueles alunos que tiveram escores baixos na eficácia profissional e escores altos na exaustão emocional e descrença ou cinismo foram considerados acometidos pela SB. 
PREVALÊNCIA DE BURNOUT ENTRE UNIVERSITÁRIOS

Quadro 2.

Prevalências e fatores associados à Síndrome de Burnout entre os universitários analisados pelos estudos incluídos nesta revisão.

\begin{tabular}{|c|c|c|c|c|}
\hline $\begin{array}{l}\text { Autor (ano de } \\
\text { publicação) }\end{array}$ & Análise estatística & Variáveis de interesse estudos & $\begin{array}{c}\text { Prevalência geral } \\
\text { (\%) e/ou Média das } \\
\text { dimensões da SB }\end{array}$ & Fatores associados \\
\hline $\begin{array}{l}\text { Carlotto, Nakamura, } \\
\text { \& Câmara, } 2006\end{array}$ & $\begin{array}{l}\text { Análises descritivas } \\
\text { Análise inferencial, } \\
\text { através da prova de } \\
\text { correlação de } \\
\text { Pearson, Teste } t \\
\text { student e ANOVA }\end{array}$ & $\begin{array}{l}\text { Sexo, idade, estado civil, presença de filhos, semestre } \\
\text { atual, número de disciplinas cursadas, ano de início do } \\
\text { curso, experiência profissional na área saúde, se possui } \\
\text { ou não outro curso superior, ocupação atual, estar } \\
\text { satisfeito com o curso, pensar em desistir do curso, } \\
\text { possuir atividade específica de lazer. }\end{array}$ & $\begin{array}{l}\text { Média } \\
\text { EE: } 2,88 \\
\text { DE: } 1,40 \\
\text { EP: } 4,90\end{array}$ & $\begin{array}{l}\text { - } \quad \text { Filhos } \\
\text { EE: } p=0,000 \\
\text { - Possuir outro curso superior } \\
\text { EE: } p=0,000 \\
\text { DE: } p=0,002 \\
\text { EP: } p=0,002 \\
\text { - Satisfação com o curso } \\
\text { EE: } p=0,000 \\
\text { DE: } p=0,002 \\
\text { EP: } p=0,003 \\
\text { Pensar em desistir } \\
\text { EE: } p=0,000 \\
\text { DE: } p=0,002 \\
\text { EP: } p=0,000\end{array}$ \\
\hline $\begin{array}{l}\text { Carlotto, } \\
\text { Câmara, Otto, } \\
\text { \& Kauffmann } \\
2010 \\
\end{array}$ & $\begin{array}{l}\text { Análises descritivas } \\
\text { Correlação de } \\
\text { Pearson }\end{array}$ & $\begin{array}{l}\text { Sexo, idade, estado civil, ter filhos, trabalhar } \\
\text { atualmente, dimensões de coping }\end{array}$ & $\begin{array}{l}\text { Média } \\
\text { EE: } 2,50 \\
\text { DE: } 1,20 \\
\text { EP: } 5,14\end{array}$ & $\begin{array}{l}\text { - EE e DE } \\
\text { Estratégias de Planejamento e } \\
\text { Reinterpretação Positiva } p<0,05 \\
\end{array}$ \\
\hline $\begin{array}{c}\text { Estela-Villa, } \\
\text { Jiménez-Román, } \\
\text { Landeo-Gutiérrez, } \\
\text { Tomateo-Torvisco, } \\
\text { \& Vega- } \\
\text { Dienstmaier, } 2010 \\
\end{array}$ & $\begin{array}{l}\text { Teste Qui- } \\
\text { quadrado } \\
\text { Teste t de student }\end{array}$ & $\begin{array}{l}\text { Sexo, estado civil, relações sentimentais, filhos, com } \\
\text { quem mora, atividade extracurricular, consumo de } \\
\text { bebida alcoólica, consumo de tabaco, salario, horas de } \\
\text { trabalho, cursos desaprovados, horas de estudo } \\
\text { semanais, horas de sono, distúrbios mentais, relação } \\
\text { em grupo. }\end{array}$ & $\begin{array}{l}\text { SB geral: } 57,2 \% \\
\text { Média } \\
\text { EE: } 67 \% \\
\text { DE: } 68,9 \% \\
\text { EP: } 35 \% \\
\end{array}$ & $\begin{array}{l}\text { - } \\
\text { EE e DE } \\
\text { Distúrbios mentais } \mathrm{p}=0,022 \\
\text { EE } \\
\text { ausência de atividades } \\
\text { extracurricular } \mathrm{p}=0,006\end{array}$ \\
\hline $\begin{array}{c}\text { Schulke, Tarouco, } \\
\text { Aloísio, \& Carlotto, } \\
2011\end{array}$ & $\begin{array}{l}\text { Análise inferencial } \\
\text { através da prova de } \\
\text { correlação de } \\
\text { Pearson e Teste } t \\
\text { de Student }\end{array}$ & $\begin{array}{c}\text { Sexo, filhos, experiência profissional, estado civil, } \\
\text { ocupação atual, semestre cursado, realização de outro } \\
\text { estagio, pratica de atividade física ou de lazer, desejo } \\
\text { de trocar ou abandonar o curso }\end{array}$ & $\begin{array}{l}\text { Média } \\
\text { EE: } 3,38 \\
\text { DE: } 1,64 \\
\text { EP: } 4,85\end{array}$ & $\begin{array}{ll}\text { - } & \mathbf{E P} \\
& \text { Praticar atividade física ou de } \\
\text { lazer } \mathrm{p}=0,030 \\
\text { realizar outros estágios } \mathrm{p}=0,046 \\
\text { DE } \\
\text { - } \\
\text { Trocar ou abandonar o curso } \\
\mathrm{p}=0,001\end{array}$ \\
\hline
\end{tabular}


Gabrielle Moura, Maria Brito, Lucinéia Pinho, Viviane Reis, Luiz Souza, \& Tatiana Magalhães

\begin{tabular}{|c|c|c|c|c|}
\hline $\begin{array}{l}\text { Campos, Jordani, } \\
\text { Zucoloto, Bonafé, \& } \\
\text { Maroco, } 2012\end{array}$ & $\begin{array}{c}\text { Análise de } \\
\text { Variância } \\
\text { Multivariada } \\
\text { (MANOVA) } \\
\text { seguida de Análise } \\
\text { de Variância } \\
\text { (ANOVA) e testes } \\
\text { post-hoc de Tukey }\end{array}$ & $\begin{array}{l}\text { Ano cursado, professor particular, ordem de } \\
\text { preferência pelo curso, expectativas iniciais em relação } \\
\text { ao curso, desempenho no curso, competência dos } \\
\text { professores, condições materiais da escola, com quem } \\
\text { vive, quem financia seus estudos, já pensou em desistir } \\
\text { do curso, já precisou tomar algum tipo de medicação } \\
\text { devido aos estudos }\end{array}$ & $\begin{array}{l}\text { SB geral: } 17 \% \\
\text { Média } \\
\text { EE: } 0,84 \\
\text { DE: } 0,71 \\
\text { EP: } 0,65\end{array}$ & $\begin{array}{l}\text { - } \text { Ordem de preferência pelo curso } \\
\mathrm{p}=0,01 \\
\text { - } \quad \text { Expectativas iniciais em relação } \\
\text { ao curso } \mathrm{p}=0,01 \\
\text { - } \quad \text { Desempenho no curso } \mathrm{p}=0,01 \\
\text { - } \quad \text { Competência dos professores } \\
\mathrm{p}=0,01 \\
\text { - } \quad \mathrm{Já} \text { pensou em desistir do curso } \\
\mathrm{p}=0,01 \\
\text { - } \quad \begin{array}{l}\text { Já precisou tomar algum tipo de } \\
\text { medicação devido aos estudos } \\
\mathrm{p}=0,04\end{array}\end{array}$ \\
\hline $\begin{array}{l}\text { Costa. Santos, } \\
\text { Santos, Melo, \& } \\
\text { Andrade, } 2012\end{array}$ & $\begin{array}{l}\text { O desvio padrão foi } \\
\text { aplicado a } \\
\text { porcentagens e } \\
\text { variáveis } \\
\text { quantitativas e } \\
\text { numéricas. } \\
\text { Associações entre } \\
\text { variáveis Odds } \\
\text { (OR) racional bruto } \\
\text { e ajustado }\end{array}$ & $\begin{array}{c}\text { Idade, sexo, relacionamento, com quem vive, religião, } \\
\text { ter parentes profissionais da saúde, renda familiar, } \\
\text { satisfação com o curso, vontade de desistir do curso, } \\
\text { baixa expectativa com o curso, falta de confiança com } \\
\text { a profissão. }\end{array}$ & $\begin{array}{l}\text { SB geral: } 10,4 \% \\
\text { EE: } 62,6 \% \\
\text { DE: } 47,4 \% \\
\text { EP: } 17,3 \%\end{array}$ & $\begin{array}{l}\text { - } \quad \text { Gênero masculino } \mathrm{p}=0,045 \\
\text { - Satisfação com a escolha de } \\
\text { carreira } \mathrm{p}=0.0002 \\
\text { - } \quad \text { Vontade de desistir do curso } \mathrm{p}= \\
0,01 \\
\text { - } \\
\text { Aquisição de habilidades } \mathrm{p}= \\
0.00001 \\
\text { - Satisfação com estratégias de } \\
\text { ensino } \mathrm{p}=0,01 \\
\text { - Sentimentos sobre atividades do } \\
\text { curso } \mathrm{p}=0.00001 \\
\text { - Curso como fonte de prazer } \mathrm{p}= \\
0.00001 \\
\text { - Baixa expectativa com o curso } \\
\mathrm{p}=0.00004\end{array}$ \\
\hline Fogaça et al., 2012 & $\begin{array}{l}\text { Teste de Mann- } \\
\text { Whitney } \\
\text { Análises de } \\
\text { Variância } \\
\text { (ANOVA) } \\
\text { Teste t Student }\end{array}$ & Período em que estuda, ano cursado. & $\begin{array}{l}\text { Média } \\
\text { EE: } 86,66 \\
\text { DE: } 79,87 \\
\text { EP: } 52,88\end{array}$ & $\begin{array}{l}\text { - Ano cursado (estudantes do } 5^{\circ} \\
\text { ano) nas três dimensões da } \mathrm{SB} \text { p }= \\
0,00\end{array}$ \\
\hline
\end{tabular}


PREVALÊNCIA DE BURNOUT ENTRE UNIVERSITÁRIOS

\begin{tabular}{|c|c|c|c|c|}
\hline $\begin{array}{l}\text { Mori, Valentell, \& } \\
\text { Nascimento, } 2012\end{array}$ & $\begin{array}{l}\text { Média, desvio } \\
\text { padrão } \\
\text { Teste Qui-quadrado }\end{array}$ & Período cursado, curso, disciplinas, notas dos alunos. & $\begin{array}{l}\text { Média } \\
\text { EE: } 3,42 \\
\text { DE: } 1,75 \\
\text { EP: } 4,00\end{array}$ & $\begin{array}{l}\text { - Período do curso } \mathrm{p}<0,05 \\
\text { - Disciplinas (bases morfológicas - } \\
\mathrm{p}=0,02 \text {; bases fisiológicas - } \mathrm{p}= \\
0,03)\end{array}$ \\
\hline $\begin{array}{l}\text { Vilela, Pacheco, \& } \\
\text { Carlos, } 2013\end{array}$ & $\begin{array}{l}\text { Estatística } \\
\text { descritiva }\end{array}$ & $\begin{array}{l}\text { Idade, sexo, estado civil, estresse, insatisfação com o } \\
\text { curso, preocupação com a renda mensal, adaptação a } \\
\text { rotina da universidade, prejuízos físicos ou mentais, } \\
\text { satisfação interpessoal com os colegas e professores, } \\
\text { preocupação com as práticas nos estágios, preocupação } \\
\text { com o mercado de trabalho. }\end{array}$ & 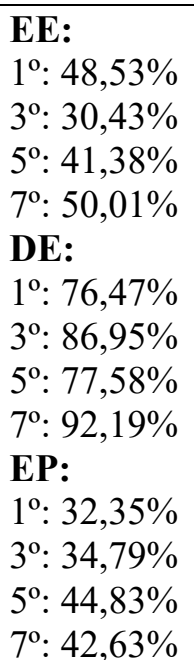 & $\begin{array}{l}\text { - Não foi realizada análise de } \\
\text { associação }\end{array}$ \\
\hline $\begin{array}{c}\text { Tomaschewski- } \\
\text { Barlem et al., } 2014\end{array}$ & $\begin{array}{l}\text { Utilização de } \\
\text { médias e } \\
\text { distribuição de } \\
\text { frequência dos } \\
\text { construtos e } \\
\text { Análises de } \\
\text { Variância } \\
\text { (ANOVA) }\end{array}$ & $\begin{array}{l}\text { Idade, se trabalha, realiza atividade de lazer, serie } \\
\text { atual, realiza atividade extracurricular, intensão de } \\
\text { desistir do curso. }\end{array}$ & $\begin{array}{l}\text { Média } \\
\text { EE: } 4,0 \\
\text { DE: } 1,8 \\
\text { EP: } 4,45\end{array}$ & $\begin{array}{l}\text { - } \quad \text { Idade } \\
\text { EP: } p=0,042 \\
\bullet \quad \text { Trabalha } \\
\text { EE: } p=0,010 \\
\text { DE: } p=0,014 \\
\text { EP: } p=0,026 \\
\bullet \quad \text { Realiza atividade de lazer } \\
\text { EP: } p=0,009 \\
\bullet \quad \text { Serie atual } \\
\text { EP: } p=0,050 \\
\text { EP: } \quad \text { Atividades extracurriculares } \\
\text { - Intenção de desistir do curso } \\
\text { DE: } p=0,005 \\
\text { EP: } p=0,016\end{array}$ \\
\hline
\end{tabular}


Gabrielle Moura, Maria Brito, Lucinéia Pinho, Viviane Reis, Luiz Souza, \& Tatiana Magalhães

\begin{tabular}{|c|c|c|c|c|}
\hline Picasso-Pozo, 2015 & Teste Qui-quadrado & $\begin{array}{l}\text { Sexo, estado civil, com quem mora, estuda e trabalha, } \\
\text { religião, prática de atividade física, doenças } \\
\text { fisiológicas. }\end{array}$ & $\begin{array}{l}\text { Níveis SB } \\
\text { Leve: } 45,7 \% \\
\text { Moderado: } 23,6 \% \\
\text { Severo: } 30,7 \% \\
\text { EE: } 45 \% \\
\text { DE: } 41,4 \% \\
\text { EP: } 45,7 \%\end{array}$ & $\begin{array}{l}\text { - } \text { Gênero } \mathrm{p}=0,044 \\
\text { - }\end{array}$ \\
\hline Gomez et al., 2015 & $\begin{array}{l}\text { Análise de cluster e } \\
\text { análise de } \\
\text { conglomerado não } \\
\text { hierárquico } \\
\text { ANOVA de um } \\
\text { fator }\end{array}$ & $\begin{array}{l}\text { Idade, sexo, nacionalidade, universidade, religião, } \\
\text { compromisso acadêmico. }\end{array}$ & $\begin{array}{l}\text { Média } \\
\text { EE: } 19,25 \\
\text { DE: } 8,34 \\
\text { EP: } 11,56\end{array}$ & • $\quad * *$ \\
\hline $\begin{array}{l}\text { Oliveira, Zucoloto, } \\
\text { \& Campos, } 2015\end{array}$ & $\begin{array}{c}\text { Desvio padrão, } \\
\text { média, frequência } \\
\text { absoluta e relativa } \\
\text { Análise de } \\
\text { Variância (Teste de } \\
\text { Kolmogorov- } \\
\text { Smirnov - p }>0,05 \text { ) } \\
\text { e } \\
\text { homocedasticidade. } \\
\text { (Teste de Levene } \\
\text { p }>0,05 \text { ), nível de } \\
\text { significância de } \\
5 \% \text {. }\end{array}$ & $\begin{array}{l}\text { Sexo, ano do curso, turno das aulas, escolha do curso, } \\
\text { expectativas iniciais em relação ao curso, classificação } \\
\text { dos professores, moradia, financiamento dos estudos, } \\
\text { uso de medicações, pensamento de desistir do curso, } \\
\text { classe econômica. }\end{array}$ & $\begin{array}{l}\text { SB geral: } 2,5 \% \\
\text { Média } \\
\text { EE: } 3,3 \\
\text { DE: } 2,18 \\
\text { EP: } 3,77\end{array}$ & $\begin{array}{l}\text { EE } \text { e DE } \\
\text { - } \quad \text { Ano cursado } p<0,01 \\
\text { - } \quad \text { Período } p<0,01 \\
\text { - } \quad \text { Expectativas com o curso } p<0,01 \\
\text { - } \quad \text { Classificação dos professores } \\
\quad p<0,01 \\
\text { EE } \\
\text { - } \quad \text { Exaustão/nunca pensaram em } \\
\text { EP desistir do curso ( } p<0,01) . \\
\text { - Período integral /eficácia } \\
\quad \text { profissional }(p<0,01) . \\
\end{array}$ \\
\hline $\begin{array}{l}\text { Chagas, Junior, } \\
\text { Cunha, Caixeta, \& } \\
\text { Fonsec, } 2016\end{array}$ & $\begin{array}{l}\text { Teste Qui- } \\
\text { Quadrado } \\
\text { Teste t para } \\
\text { amostras } \\
\text { independentes. }\end{array}$ & $\begin{array}{l}\text { Faixa etária, sexo, período, tipo de residência, estado } \\
\text { civil, trabalho e fatores estressores. }\end{array}$ & $\begin{array}{l}\text { SB geral: } 11,4 \% \\
\text { Média: } \\
\text { EE: } 4,32 \\
\text { DE: } 2,96 \\
\text { EP: } 5,36\end{array}$ & $\begin{array}{l}\text { - Fardo físico e emocional imposto } \\
\text { pelo curso } \mathrm{p}=0,041 \\
\text { - } \\
\text { Realizar provas e trabalhos } \mathrm{p}= \\
\text { - } \\
\text { Excessiva carga de estudo } \\
\mathrm{p}=0,014 \\
\text { - } \\
\text { - } \quad \text { Couco preparo teórico } \mathrm{p}=0,014 \\
\text { - } \quad \text { Falta de restudo e lazer } \mathrm{p}=0,006 \\
\mathrm{p}=0,002\end{array}$ \\
\hline
\end{tabular}


PREVALÊNCIA DE BURNOUT ENTRE UNIVERSITÁRIOS

\begin{tabular}{|c|c|c|c|l|}
\hline & & & & $\begin{array}{l}\text { Dificuldade de adaptação } \\
\mathrm{p}=0,035 \\
\text { Adquirir material de estudo } \\
\mathrm{p}=0,031 \\
\text { Preocupação quanto a colocação } \\
\text { profissional } \mathrm{p}=0,021\end{array}$ \\
\hline $\begin{array}{c}\text { Almeida, Souza, } \\
\text { Almeida, Almeida, } \\
\text { \& Almeida, 2016 }\end{array}$ & $\begin{array}{c}\text { Teste Qui- } \\
\text { Quadrado } \\
\text { Análises bivariadas } \\
\text { Regressão logística. }\end{array}$ & $\begin{array}{c}\text { Idade, sexo, estado civil, atividades extracurriculares, } \\
\text { pensar em abandonar o curso, situação financeira, } \\
\text { reprovação em exames }\end{array}$ & $\begin{array}{l}\text { SB geral: } 14,9 \% \\
\text { Risco SB: } 57,7 \% \\
\text { Ausência SB: } 27,4 \%\end{array}$ & $\begin{array}{l}\text { Pensar em abandonar o curso } \\
\mathrm{p}<0,001 \\
\text { Reprovação } \mathrm{p}=0,047\end{array}$ \\
\hline
\end{tabular}

**Não informado pelos autores

EE: exaustão emocional; DE: descrença; EP: eficácia profissional. 
Em relação aos fatores associados, os artigos aqui incluídos destacaram: sexo, idade, tipo de moradia, falta de tempo de lazer, inscrição em um maior número de disciplinas, insatisfação com o curso, intenção de sair do curso, semestres avançados, atendimento complementar, consumo de medicações devido aos estudos.

Para facilitar a visualização, foi criado um quadro (Quadro 3) com as principais conclusões apontadas pelos estudos, evidenciando que a vida acadêmica, relacionada, em geral, à insatisfação e vontade de desistir do curso, como principais fatores associados à Síndrome de Burnout entre os universitários.

\section{Quadro 3.}

Principais conclusões dos artigos incluídos na revisão, 2006 a 2017.

\begin{tabular}{|c|c|}
\hline $\begin{array}{l}\text { Autor (ano de } \\
\text { publicação) }\end{array}$ & Principais conclusões \\
\hline $\begin{array}{l}\text { Carlotto, } \\
\text { Nakamura, \& } \\
\text { Câmara, 2006 }\end{array}$ & $\begin{array}{l}\text { "A Exaustão Emocional evidencia índice médio mais elevado, o que pode ser um } \\
\text { possível indicativo de Burnout no futuro, visto que provavelmente está sendo contida } \\
\text { pelo alto índice de Eficácia Profissional do grupo". }\end{array}$ \\
\hline $\begin{array}{l}\text { Carlotto, } \\
\text { Câmara, Otto, \& } \\
\text { Kauffmann, } \\
2010\end{array}$ & $\begin{array}{l}\text { "Verificou-se que o não pensar sobre alternativas para lidar com um estressor através } \\
\text { de estratégias de ação e não reinterpretar uma situação negativa ou tensa em termos } \\
\text { positivos aumenta o sentimento de desgaste decorrente das atividades acadêmicas e, } \\
\text { consequentemente, o aluno se torna mais descrente em relação ao que sua formação } \\
\text { pode lhe proporcionar". }\end{array}$ \\
\hline $\begin{array}{l}\text { Estela-Villa et } \\
\text { al., } 2010\end{array}$ & $\begin{array}{l}\text { "Alta prevalência de SB entre os estudantes de medicina da } 7^{\text {a }} \text { série, onde } \\
\text { aproximadamente } 6 \text { em cada } 10 \text { atendem aos critérios do SB". }\end{array}$ \\
\hline $\begin{array}{l}\text { Schulke et al., } \\
2011\end{array}$ & $\begin{array}{l}\text { "Observou-se um índice médio de Exaustão Emocional, um possível fator de risco de } \\
\text { desenvolvimento de Burnout no futuro profissional. Neste momento, tal situação pode } \\
\text { estar sendo contida pelo alto índice de Eficácia Profissional e confiança na } \\
\text { aprendizagem adquirida". }\end{array}$ \\
\hline $\begin{array}{l}\text { Campos et al., } \\
2012\end{array}$ & $\begin{array}{l}\text { "A prevalência da Síndrome de Burnout entre os estudantes de Odontologia foi de } \\
17 \% \text {, com fatores associados relacionados a satisfação e vontade de desistir do curso". }\end{array}$ \\
\hline $\begin{array}{l}\text { Costa et al., } \\
2012\end{array}$ & $\begin{array}{l}\text { "Observou-se que } 10,3 \% \text { dos alunos atendem aos critérios de diagnóstico } \\
\text { tridimensional da síndrome de Burnout". Estiveram associados à SB a confiança em } \\
\text { suas habilidades clínicas }(\mathrm{OR}=6,47) \text {, aqueles que se sentiam desconfortáveis com as } \\
\text { atividades do curso }(\mathrm{OR}=5,76) \text {, e aqueles que não viram o curso como fonte de prazer } \\
(\mathrm{OR}=4,68) \text {. "Acreditamos que esse Burnout ocorre quando os alunos começam o } \\
\text { contato direto com os pacientes porque os alunos experimentam um tempo de } \\
\text { transição, incerteza e maior responsabilidade". }\end{array}$ \\
\hline $\begin{array}{l}\text { Fogaça et al., } \\
2012\end{array}$ & $\begin{array}{l}\text { "Os Resultados obtidos na avaliação dos estudantes de } 5^{\circ} \text { ano, de ambos os períodos, } \\
\text { sugerem a presença da SB, levando em consideração que os escores nas dimensões de } \\
\text { exaustão e descrença são estatisticamente significativos". } \\
\text { "Os resultados obtidos [...] descrevem - entre os estudantes - uma variedade de } \\
\text { estressores semelhantes aos encontrados em trabalhadores de alto desempenho laboral, } \\
\text { pois o processo de aprendizagem exige [..] uma atualização constante, podendo gerar } \\
\text { competitividade, conflitos e potenciais estressores, levando-os a problemas } \\
\text { emocionais". }\end{array}$ \\
\hline $\begin{array}{l}\text { Mori, Valentell, } \\
\& \text { Nascimento, } \\
2012\end{array}$ & $\begin{array}{l}\text { "A implantação da reforma curricular não foi um fator de proteção para a Síndrome de } \\
\text { Burnout. Houve também associação entre a síndrome e o rendimento acadêmico dos } \\
\text { estudantes em disciplinas bases morfológicas e fisiologia da primeira e da segunda } \\
\text { série". }\end{array}$ \\
\hline $\begin{array}{l}\text { Vilela, Pacheco, } \\
\& \text { Carlos, } 2013\end{array}$ & $\begin{array}{l}\text { "Observou-se que } 78 \% \text { de todos os alunos estudados, apresentavam sentimento de } \\
\text { estresse e alguma insatisfação com o curso de Enfermagem no momento da pesquisa". }\end{array}$ \\
\hline
\end{tabular}




\section{PREVALÊNCIA DE BURNOUT ENTRE UNIVERSITÁRIOS}

\begin{tabular}{|l|l|}
\hline $\begin{array}{l}\text { Tomaschewski- } \\
\text { Barlem et al., } \\
2014\end{array}$ & $\begin{array}{l}\text { "Observou-se que o processo de Burnout pode estar em desenvolvimento nesses } \\
\text { estudantes investigados". }\end{array}$ \\
\hline $\begin{array}{l}\text { Picasso-Pozo et } \\
\text { al., } 2015\end{array}$ & $\begin{array}{l}\text { "Verificou-se que 30,7\% dos estudantes apresentaram nível severo da SB, 45\% da } \\
\text { amostra com alto nível de exaustão emocional, 41,4\% um alto nível de cinismo e } \\
45,7 \% \text { apresentaram baixo nível de eficácia acadêmica. }\end{array}$ \\
\hline $\begin{array}{l}\text { Gomez et al., } \\
2015\end{array}$ & $\begin{array}{l}\text { "O desempenho acadêmico está associado ao nível de engajamento com estudos, mas } \\
\text { não com Burnout". }\end{array}$ \\
\hline $\begin{array}{l}\text { Oliveira, } \\
\text { Zucoloto, \& } \\
\text { Campos, 2015 }\end{array}$ & $\begin{array}{l}\text { "As variáveis de pensamento de desistir do curso, ano, turno, expectativas iniciais e } \\
\text { classificação dos professores apresentaram relação significativa com os escores médios } \\
\text { dos fatores da Síndrome de Burnout". }\end{array}$ \\
\hline $\begin{array}{l}\text { Chagas et al., } \\
2016\end{array}$ & $\begin{array}{l}\text { "Observou-se uma ampla rede de fatores que contribuem para o desenvolvimento da } \\
\text { síndrome de Burnout, com uma ocorrência de 11,4\% em estudantes de medicina". }\end{array}$ \\
\hline $\begin{array}{l}\text { Almeida et al., } \\
2016\end{array}$ & $\begin{array}{l}\text { "Em geral, estudos realizados em outros países mostram uma maior prevalência de SB } \\
\text { do que aqueles no Brasil. No presente estudo, 14,9\% dos alunos tinham SB, associado } \\
\text { em pensar em desistir do curso e ter sido reprovado". }\end{array}$ \\
\hline
\end{tabular}

\section{DISCUSSÃO}

Este estudo avaliou a SB em acadêmicos, na tentativa de melhor conhecer sobre os fatores associados à síndrome em vários contextos culturais. Para isso, utilizou-se como critério o emprego do instrumento MBI-SS como padrão-ouro, uma vez que possuiu confiabilidade e validade atestada em diferentes países como Brasil, Peru, Chile, Portugal, Coreia, China, Estados Unidos (Campos et al., 2013; Estela-Villa et al., 2010).

Apesar da sua validação, o MBI-SS possui itens que, em determinados lugares, devem passar por modificações para atenderem a população local. Pesquisa realizada com alunos chineses mostra que as questões 4 e 13 da exaustão emocional apresentam carga de fator relativamente baixa por apresentar uma ambivalência semântica, reforçando a necessidade de adaptar e estudar a SB em vários contextos (Hu \& Schaufeli, 2009).

Ao analisar os artigos incluídos nesta revisão, pode-se observar que são necessários altos escores em exaustão e descrença e baixos escores em eficácia profissional para indicar a presença da SB em acadêmicos (Schaufeli, Martinez, Pinto, Salanova, \& Bakker 2002). Seguindo estes critérios, os estudos mostraram prevalência que chegou a 57,2\% em estudantes de Lima, Peru (Estela-Villa et al., 2010). Ao observar prevalência que excede metade da amostra analisada, destaca-se o impacto que este agravo possa acarretar na vida dos universitários, podendo sofrer lacunas em sua formação acadêmica, além dos possíveis danos psíquicos.

Os autores do estudo em Lima (Peru) explicam que a alta frequência da SB entre os acadêmicos investigados pode ser explicada pela falta de serviços psicológicos para acompanhamento acadêmico nas universidades peruanas, além da falta de aperfeiçoamento do ambiente de ensino e da didática de aprendizagem (Estela-Villa et al., 2010).

A medicina e a odontologia foram os cursos com maior índice de Burnout. Autores explicam que isso ocorre pelo fato de os alunos destes cursos estarem em constante contato com pacientes, realizando procedimentos invasivos que promovem riscos a si mesmos e aos pacientes, gerando alto nível de estresse (Almeida et al., 2016; Campos et al., 2012; Goni et al.,2015).

Apesar de algumas pesquisas não apresentarem índices de SB significativos, é pertinente enfatizar que houve um aumento no número de estudantes que se encontravam nas faixas de "risco", representada pelos acadêmicos que apresentam altos escores de exaustão emocional, o que segundo o modelo de Burnout de Maslach (1976), é a primeira dimensão a surgir (Magri, Melchior, Jarina, Simonaggio, \& Bataglion, 2016; Maslach, 1976; Suhulke et al., 2011). 
Em relação aos fatores associados à SB, identificaram-se o variável sexo, idade, tipo de moradia, falta de tempo de lazer, inscrição em um maior número de disciplinas, insatisfação com o curso, intenção de sair do curso, semestres avançados, atendimento complementar, e o consumo de medicações devido aos estudos (Almeida et al., 2016; Campos et al., 2012; Costa et al., 2012; Tomaschewski-Barlem et al., 2014).

Sobre a variável "sexo", observou-se que os homens apresentaram estatísticas significativas nos escores, exibindo pontuações menores para a eficácia profissional e para a exaustão emocional (Campos et al., 2012; Hu \& Schaufeli, 2009). Um estudo longitudinal (Magri et al., 2016) com alunos de odontologia mostrou que as mulheres estão predominantemente na zona de risco da SB, observando-se diferença entre os gêneros para a exaustão emocional durante três anos $(p=0,002 ; p$ $=0,003 ; p=0,01$, respectivamente). Estes mesmos autores associam os achados ao fato de as universitárias encararem as pressões e responsabilidades atribuídas pelo curso com elevado desgaste emocional (Magri et al., 2016).

Alunos dos primeiros períodos mostraram-se com escores de exaustão e de descrença significativamente maiores, o que pode ser explicado pela imaturidade com que o mesmo ingressa na graduação. Já em relação às disciplinas, evidenciou-se que quanto maior o número de matérias em curso, maior é o sentimento de desgaste decorrente do ensino devido à sobrecarga de matérias, exercícios e provas (Campos et al., 2012; Carlotto, Nakamura \& Câmara, 2006; TomaschewskiBarlem et al., 2014).

Outros fatores significativos foi o fato de que os alunos que desejam desistir do curso apresentaram maiores sentimentos de descrença e exaustão, e os que associavam o estudo com o trabalho se percebiam menos eficazes. Estes achados podem ser explicados tendo em vista que ao não acreditarem nos resultados de sua formação, os alunos tornam-se mais descrentes ao realizarem suas atividades (Oliveira, Zucoloto, \& Campos, 2015; Schulke et al., 2011; Tomaschewski-Barlem et al., 2014).

Desta forma, a insatisfação sugere que o aluno, ao estar descontente com a escolha do curso, realiza suas atividades sem vontade, o que torna as tarefas mais desgastantes e propicia uma atitude de indiferença e impessoalidade que culminam no ceticismo. Constatou-se que a insatisfação com o curso aumenta progressivamente e se relaciona às estratégias de ensino, podendo, ainda, estar associada ao modelo de educação, incluindo a alta carga horária. Percebe-se, portanto, que a insatisfação referida não está relacionada à profissão em si, mas sim à dinâmica pedagógica do curso (Carlotto, Nakamura, \& Câmara, 2006; Costa et al., 2012; Vilela et al., 2013).

Outra variável que se manteve associada em alguns dos estudos analisados aqui foi o "consumo de medicações devido aos estudos", destacando as medicações para melhoria do rendimento escolar. Dentre as drogas mais utilizadas no meio acadêmico, têm-se as anfetaminas, que tem seu uso cada vez mais frequente e irregular. Os efeitos adversos causados por estas medicações envolvem fadiga, dificuldade de concentração, anorexia, apatia, insônia, irritabilidade e depressão. Tais efeitos podem ter tanto um impacto social considerável, uma vez que na ocorrência destes, aumenta-se a chance do deterioramento da saúde do indivíduo, propiciando o desenvolvimento de sintomatologias depressivas e afetando as relações interpessoais e execução de tarefas rotineiras, podendo ser um fator importante nos aspectos envolvidos na SB (Osorio, 2013; Takitane et al., 2013).

Observou-se, também, que "como" e "com quem" os alunos moram podem ser fatores preventivos ou de risco no desenvolvimento da SB, visto que os que moravam sozinhos, longe dos pais e familiares, possuíam índices maiores de exaustão emocional, assim como os que possuem financiamento para custear os estudos, demonstrando que o apoio da família e amigos, tanto no quesito emocional como financeiro, são fatores protetores na ocorrência da SB (Christofoletti et al., 2007; Goni et al., 2015). 


\section{PREVALÊNCIA DE BURNOUT ENTRE UNIVERSITÁRIOS}

Como limitações envolvendo os estudos incluídos nesta revisão, destacam-se o fato de todos os estudos serem transversais, não permitindo inferir um nexo causal entre a síndrome e as condições acadêmicas dos universitários. Outra limitação seria o viés de publicação, uma vez que alguns estudos não são publicados em razão dos resultados obtidos (ausência de resultados estatisticamente significativos, por exemplo) ou, ainda, estudos que não foram encontrados por não estarem publicados em periódicos indexados nas bases investigadas. Torna-se necessário reconhecer a possibilidade de viés de interpretação, já que a revisão da literatura se configura como um trabalho de interpretação de dados.

Os resultados encontrados nesta revisão indicam uma ascensão da pesquisa epidemiológica referente à SB em estudantes universitários, revelando um aumento da prevalência nos últimos anos, independentemente dos instrumentos utilizados, mesmo ainda que esta apresente dificuldade em comparar os dados encontrados, pois o instrumento permite os resultados em médias ou porcentagens.

Notou-se que a prevalência máxima da SB identificado foi significativa. Além disso, nos estudos que analisaram as dimensões da $\mathrm{SB}$, os valores excederam a metade das amostras investigadas.

Os fatores associados estiveram, em sua maioria, envolvidos com questões diretamente relacionadas à vida acadêmica, destacando a falta de tempo de lazer, inscrição em um maior número de disciplinas, insatisfação com o curso, intenção de sair do curso, semestres avançados, atendimento complementar, consumo de medicações devido aos estudos. Desta forma, torna-se importante a criação de estratégias de escuta e acolhimento dos universitários, com ações que possibilitem o desenvolvimento de atividades que dêem prazer durante a graduação. Contudo, fica clara a necessidade de estudos longitudinais para confirmar as tendências encontradas a esse respeito.

Apesar de a SB ser analisada com muita frequência em grupos de trabalhadores já inseridos no mercado de trabalho, considerar os universitários como um grupo importante dentro do contexto ocupacional, uma vez que o início do Burnout pode ocorrer no período acadêmico e persistir na vida profissional. O que gera repercussões sintomatológicas psicossomáticas e comportamentais, bem como proporcionar redução na qualidade da assistência prestada aos clientes/pacientes/usuários, tanto durante a graduação, quanto na vida profissional.

Neste sentindo, são necessárias novas pesquisas que explorem essa temática em diferentes regiões e nos vários contextos culturais e de formação, a fim de prover dados e informações suficientes para que as Instituições de Ensino Superior possam prever impedir ou cessar as manifestações e prejuízos da SB em seus alunos.

\section{REFERÊNCIAS}

Almeida, G. D. C., Souza, H. R. D., Almeida, P. C. D., Almeida, B. D. C., \& Almeida, G. H. (2016). The prevalence of burnout syndrome in medical students. Archives of Clinical Psychiatry (São Paulo), 43(1), 6-10. doi:10.1590/0101-60830000000072

Araújo, D. M. R., Vilarim, M. M., Sabroza, A. R., \& Nardi, A. E. (2010). Depressão no período gestacional e baixo peso ao nascer: uma revisão sistemática da literatura. Cadernos de Saúde Pública, 26, 219-227. doi:10.1590/S0102-311X2010000200002

Azevedo Guido, L., da Silva, R. M., Goulart, C. T., de Oliveira Bolzan, M. E., \& Lopes, L. F. D. (2012). Síndrome de Burnout em residentes multiprofissionais de uma universidade pública. Revista da Escola de Enfermagem da USP, 46(6), 1477-1483. doi:10.1590/S008062342012000600027

Campos, J. A. D. B., Jordani, P. C., Zucoloto, M. L., Bonafé, F. S. S., \& Maroco, J. (2013). Burnout in dental students: effectiveness of different methods. Revista de Odontologia da UNESP, 42(5), 324-329. doi:10.1590/S1807-25772013000500002 
Campos, J. A. D. B., Jordani, P. C., Zucoloto, M. L., Bonafé, F. S. S., \& Maroco, J. (2012). Síndrome de Burnout em graduandos de Odontologia. Revista Brasileira de Epidemiologia, 15, 155-165. doi:10.1590/S1415-790X2012000100014

Carlotto, M. S., Nakamura, A. P., \& Câmara, S. G. (2006). Síndrome de Burnout em estudantes universitários da área da saúde. Psico, 37(1), 57-62.

Carlotto, M. S., Câmara, S. G., Otto, F., \& Kauffmann, P. (2010). Síndrome de burnout e coping em estudantes de psicologia. Boletim de psicologia, 59(131), 167-178.

Chagas, M. K. S., Junior, D. D. B. M., Cunha, G. N., Caixeta, R. P., \& Fonseca, E. F. (2016). Ocorrência da Síndrome de Burnout em acadêmicos de medicina de instituição de ensino no interior de Minas Gerais. Revista de Medicina e Saúde de Brasília, 5(2).

Christofoletti, G., Trelha, C. S., Galera, R. M., \& Feracin, M. A. (2007). Síndrome de burnout em acadêmicos de fisioterapia. Fisioterapia e pesquisa, 14(2), 35-39. doi: 10.1590/fpusp.v14i2.75857

Costa, E. F. D. O., Santos, S. A., Santos, A. T. R. D. A., Melo, E. V. D., \& Andrade, T. M. D. (2012). Burnout Syndrome and associated factors among medical students: a cross-sectional study. Clinics, 67(6), 573-580. doi: 10.6061/clinics/2012(06)05

Downs, S. H., \& Black, N. (1998). The feasibility of creating a checklist for the assessment of the methodological quality both of randomised and non-randomised studies of health care interventions. Journal of Epidemiology \& Community Health, 52(6), 377-384. doi: 10.1136/jech.52.6.377

Estela-Villa, L. M., Jiménez-Román, C. R., Landeo-Gutiérrez, J. S., Tomateo-Torvisco, J. D., \& Vega-Dienstmaier, J. M. (2010). Prevalencia de síndrome de burnout en alumnos del séptimo año de medicina de una universidad privada de Lima, Perú. Revista de Neuro-Psiquiatría, 73(4). doi: $10.20453 /$ rnp.v73i4.1700

Fogaça, M., de Moraes Hamasaki, E. I., Barbieri, C. A. P., Borsetti, J., Martins, R. Z., Silva, I. G., \& Ribeiro, L. P. (2012). Burnout em estudantes de psicologia: diferenças entre alunos iniciantes e concluintes. Aletheia, (38-39).

Gómez, P., Pérez, C., Parra, P., Ortiz, L., Matus, O., McColl, P., ... \& Meyer, A. (2015). Relación entre el bienestar y el rendimiento académico en alumnos de primer año de medicina. Revista médica de Chile, 143(7), 930-937. doi:10.4067/S0034-98872015000700015

Goni, M., Danza, Á., Urgoiti, M., \& Durante, E. (2015). Correlación entre clima educativo y síndrome de burnout en practicantes internos de la carrera de Doctor en Medicina de Uruguay. Revista Médica del Uruguay, 31(4), 272-281.

$\mathrm{Hu}, \mathrm{Q} .$, \& Schaufeli, W. B. (2009). The factorial validity of the Maslach Burnout Inventory-student survey in China. Psychological Reports, 105(2), 394-408. doi: 10.2466/PR0.105.2.394-408

Liberati, A., Altman, D. G., Tetzlaff, J., Mulrow, C., Gøtzsche, P. C., Ioannidis, J. P., \& Moher, D. (2009). The PRISMA statement for reporting systematic reviews and meta-analyses of studies that evaluate health care interventions: explanation and elaboration. PLoS medicine, 6(7), e1000100. doi: 10.1371/journal.pmed.1000100

Magri, L. V., Melchior, M. O., Jarina, L., Simonaggio, F. F., \& Bataglion, C. (2016). Relationship between temporomandibular disorder symptoms signs and Burnout syndrome among dentistry students. Revista Dor, 17(3), 171-177. doi: 10.5935/1806-0013.20160065

Maslach, C. (1976). Burned-out. Human behavior, 5(9), 16-22.

Maslach, C., \& Jackson, S. E. (1981). The measurement of experienced burnout. Journal of organizational behavior, 2(2), 99-113. doi: 10.1002/job.4030020205

Mori, M. O., Valentell, T. C., \& Nascimento, L. F. C. (2012). Síndrome de Burnout e rendimento acadêmico em estudantes da primeira à quarta série de um curso de graduação em medicina. Revista Brasileira de Educação Médica,36(4), 5. doi: 10.1590/S010055022012000600013 


\section{PREVALÊNCIA DE BURNOUT ENTRE UNIVERSITÁRIOS}

Oliveira, V., Zucoloto, M. L., \& Campos, J. A. D. B. (2015). Síndrome de Burnout em estudantes de Farmácia-Bioquímica: um estudo transversal. Revista Brasileira de Pesquisa em Saúde/Brazilian Journal of Health Research, 17(1), 95-102. doi:10.21722/rbps.v17i1.12456

Osorio, J. H. (2013). Implicaciones metabólicas y clínicas de algunas drogas de diseño. Normas Editoriales, 110.

Peleias, I. R., Guimarães, E. R., Chan, B. L., \& Carlotto, M. S. (2017). A síndrome de Burnout em estudantes de ciências contábeis de IES Privadas: pesquisa na cidade de São Paulo. Revista de Educação e Pesquisa em Contabilidade (REPeC), 11(1). doi: 10.17524/repec.v11i1.1468

Picasso-Pozo, M. (2015). Síndrome de burnout en estudiantes de Odontología de una universidad peruana. KIRU Revista de la Facultad de Odontología-Universidad de San Martín de Porres, $9(1)$.

Ricardo, Y. R., \& Paneque, F. R. R. (2013). Burnout estudiantil universitario. Conceptualización y estudio. Salud mental, 36(4), 337-345.

Rossi, C. E., \& Vasconcelos, F. D. A. G. D. (2010). Peso ao nascer e obesidade em crianças e adolescentes: uma revisão sistemática. Revista Brasileira de Epidemiologia, 13, 246-258. doi:10.1590/S1415-790X2010000200007

Schaufeli, W. B., Martinez, I. M., Pinto, A. M., Salanova, M., \& Bakker, A. B. (2002). Burnout and engagement in university students: A cross-national study. Journal of cross-cultural psychology, 33(5), 464-481. doi:10.1177/0022022102033005003

Schulke, A. P., Tarouco, A. M., Aloísio, A. I. K., \& Carlotto, M. S. (2011). El Síndrome de Burnout en los alumnos en prácticas de fisioterapia. Diversitas: perspectivas en psicología, 7(1), $167-$ 177.

Sobrinho, J. D. (2014). Universidade e novos modos de produção, circulação e aplicação do conhecimento. Avaliação: Revista da Avaliação da Educação Superior, 19(3). doi: 10.1590/S1414-40772014000300007

Takitane, J., Oliveira, L. G. D., Endo, L. G., Oliveira, K. C. B. G. D., Muñoz, D. R., Yonamine, M., \& Leyton, V. (2013). Uso de anfetaminas por motoristas de caminhão em rodovias do Estado de São Paulo: um risco à ocorrência de acidentes de trânsito? Ciência \& Saúde Coletiva, 18, $1247-$ 1254. doi:10.1590/S1413-81232013000500009

Thiengo, D. L., Santos, J. F. D. C., Mason, V. C., Abelha, L., \& Lovisi, G. M. (2011). Associação entre apoio social e depressão durante a gestação: uma revisão sistemática. Caderno de Saúde Coletiva, 19(2), 129-38.

Tomaschewski-Barlem, J. G., Lerch Lunardi, V., Marcelino Ramos, A., Silva da Silveira, R., Devos Barlem, E. L., \& Mirapalheta Ernandes, C. (2013). Manifestações da síndrome de burnout entre estudantes de graduação em enfermagem. Texto \& Contexto Enfermagem, 22(3). doi: 10.1590/S0104-07072013000300023

Tomaschewski-Barlem, J. G., Lunardi, V. L., Lunardi, G. L., Barlem, E. L. D., Silveira, R. S. D., \& Vidal, D. A. S. (2014). Burnout syndrome among undergraduate nursing students at a public university. Revista latino-americana de enfermagem,22(6), 934-941. doi:10.1590/01041169.3254.2498

Vilela, S. D. C., Pacheco, A. E., \& Carlos, A. L. D. S. (2013). Síndrome de burnout e estresse em graduandos de enfermagem. Revista de enfermagem do Centro Oeste Mineiro, 3(3), 780-787. doi: 10.19175/recom.v0i0.415 